\title{
Underground mine risk assessment by using FMEA in the presence of uncertainty
}

\author{
Shahram Shariati ${ }^{\mathbf{a}^{*}}$
}

Department of Geology, Sari Branch, Islamic Azad University, Sari, Iran

\section{H R O N I C L E}

\begin{tabular}{l}
\hline Article history: \\
Received October 15, 2013 \\
Received in revised format \\
March 22014 \\
Accepted April 7, 2014 \\
Available online \\
April 82014 \\
\hline Keywords: \\
Hazard \\
Fuzzy FMEA \\
Risk Priority Number \\
Phosphate mining project
\end{tabular}

\begin{abstract}
A B S T R A C T
Managers always look for systems with minimum hazards, which cause problems for performance of projects. The largest and the most important hazards of working underground mines can be associated with health, safety and environmental Failure mode and effects analysis (FMEA) is a widely used technique to identify the potential failure modes for measuring reliability of a product or a process. FMEA is performed by developing a risk priority number (RPN), which is the product of severity, occurrence, and detection ratings. On the other hand, with regard to uncertainty in the decision-making, fuzzy theory can help model the inherent uncertainty involved in the underground mining projects. Fuzzy FMEA provides a tool that can work in a better way with vague concepts using insufficient information compared with conventional FMEA. The comparison between the results of the conventional FMEA with those of the proposed model shows that the fuzzy model has a high potential to formulate the level of risk.
\end{abstract}

\section{Introduction}

Mining is an ancient job, long recognized as being arduous and liable to injury and various kinds of diseases. The mining industry, perhaps more than others, has been plagued by different risks often resulting in poor performance with increasing expenses and time delay, even project failure. The nature of many mining projects has made it a challenging regime to handle risks, expenses, and increasing complexity on mining approaches. Mining risk analysis, more specifically at the early stages of the project, is intricate because the nature of risk is usually influenced by various factors including human error and the data and existing information. Under various circumstances, it may be difficult to evaluate the risks associated with a project due to the great uncertainty involved. Many risk assessment techniques currently implemented in the mining industry are comparatively mature, such as Fault Tree Analysis, Event Tree Analysis, Monte Carlo Analysis, Scenario Planning, Sensitivity Analysis, Failure Mode and Effects Analysis, Program Evaluation and Review Technique.

* Corresponding author.

E-mail addresses: shahramshariati62@gmail.com (S. Shariati) 
Failure mode and effects analysis (FMEA) is one of the best techniques for assessing the effect(s) of potential failure modes of subsystems, assemblies, components, or functions (Ericson, 2005). FMEA is an efficient problem prevention methodology, which easily interfaces with many engineering and reliability methods (Tay \& Lim, 2006). Nevertheless, for effective applications of these sophisticated quantitative approaches, high quality data are a prerequisite (Winch, 2002). Unfortunately, such data are hard to collect or may not exist in the underground mining industry. Moreover, they are difficult to address the existing uncertainties and subjectivities associated with construction activities. It is therefore essential to develop new risk analysis techniques to evaluate construction risks in an acceptable way where any risk information produced is processed and reliably applied to decision making in the project management (Zeng et al., 2007).

This paper focuses on the use of fuzzy inference approaches as an alternative to overcome the weaknesses associated with the traditional FMEA system. Traditional FMEA applies the risk priority number (RPN) ranking system to make an assessment on the risk level of failures, to rank failures, and to prioritize actions (Ireson et al., 1995). Comparing with traditional FMEA, the fuzzy approach allows failure risk evaluation and prioritization to be conducted based on experts’ knowledge.

\section{FMEA methodology}

Each failure mode will be assessed in three parameters, namely, severity, likelihood of occurrence, and difficulty of detection of the failure mode (Chin et al., 2008). A typical evaluation system gives a number between 1 and 10 where 1 represents the best and 10 states for the worst case for each of the three parameters. By multiplying the values for severity (S), occurrence (O), and detectability (D), the decision making team calculates a risk priority number (RPN), which is $\mathrm{RPN}=\mathrm{S} \times \mathrm{O} \times \mathrm{D}$, as described in Tables 1, 2, and 3.

\section{Table 1}

Crisp ratings for occurrence of a failure (Chin et al., 2008; Wang et al., 2009)

\begin{tabular}{ccc}
\hline Rating & Probability of occurrence & Failure probability \\
\hline 10 & Very high: failure is almost inevitable & $>1$ in 2 \\
9 & High: repeated failures & 1 in 3 \\
8 & & 1 in 8 \\
7 & Moderate: occasional failures & 1 in 20 \\
6 & & 1 in 80 \\
5 & Low: relatively few failures & 1 in 400 \\
4 & Remote: failure is unlikely & 1 in 2000 \\
3 & & 1 in 15,000 \\
1 & & $<1$ in $1,500,000$ \\
\hline
\end{tabular}

\section{Table 2}

Crisp ratings for severity of a failure (Chin et al., 2008; Wang et al., 2009)

\begin{tabular}{cll}
\hline Rating & Effect & Severity of effect \\
\hline 10 & $\begin{array}{l}\text { Hazardous without } \\
\text { warning }\end{array}$ & $\begin{array}{l}\text { Very high severity ranking when a potential failure mode effects safe system operation } \\
\text { without warning }\end{array}$ \\
\hline $\begin{array}{l}\text { Hazardous with } \\
\text { warning }\end{array}$ & $\begin{array}{l}\text { Very high severity ranking when a potential failure mode effects safe system operation } \\
\text { with warning }\end{array}$ \\
\hline 7 & Very high & System inoperable with destructive failure without compromising safety \\
6 & Moderate & System inoperable with equipment damage \\
5 & Low & System inoperable with minor damage \\
4 & Very low & System inoperable without damage \\
3 & Minor & System operable with significant degradation of performance \\
2 & Very minor & System operable with some degradation of performance \\
1 & None & System operable with minimal interference \\
\hline
\end{tabular}


Table 3

Crisp ratings for detection of a failure (Chin et al., 2008; Wang et al., 2009)

\begin{tabular}{|c|c|c|}
\hline Rating & Detection & Likelihood of detection by design control \\
\hline 10 & $\begin{array}{l}\text { Absolute } \\
\text { uncertainty }\end{array}$ & Design control cannot detect potential cause/mechanism and subsequent failure mode \\
\hline 9 & Very remote & $\begin{array}{l}\text { Very remote chance the design control will detect potential cause/mechanism and } \\
\text { subsequent failure mode }\end{array}$ \\
\hline 8 & Remote & $\begin{array}{l}\text { Remote chance the design control will detect potential cause/mechanism and subsequent } \\
\text { failure mode }\end{array}$ \\
\hline 7 & Very low & $\begin{array}{l}\text { Very low chance the design control will detect potential cause/mechanism and subsequent } \\
\text { failure mode }\end{array}$ \\
\hline 6 & Low & $\begin{array}{l}\text { Low chance the design control will detect potential cause/mechanism and subsequent } \\
\text { failure mode }\end{array}$ \\
\hline 5 & Moderate & $\begin{array}{l}\text { Moderate chance the design control will detect potential cause/mechanism and subsequent } \\
\text { failure mode }\end{array}$ \\
\hline 4 & Moderately high & $\begin{array}{l}\text { Moderately high chance the design control will detect potential cause/mechanism and } \\
\text { subsequent failure mode }\end{array}$ \\
\hline 3 & High & $\begin{array}{l}\text { High chance the design control will detect potential cause/mechanism and subsequent } \\
\text { failure mode }\end{array}$ \\
\hline 2 & Very high & $\begin{array}{l}\text { Very high chance the design control will detect potential cause/mechanism and } \\
\text { subsequent failure mode }\end{array}$ \\
\hline 1 & Almost certain & Design control will detect potential cause/mechanism and subsequent failure mode \\
\hline
\end{tabular}

The failure modes with higher RPNs are considered to be more important and will be given higher priorities for correction. The RPNs helps the decision making team detect the parts or processes, which require the priority actions for improvement or appropriate reaction and it depends entirely on the company policy. The FMEA has high capability to model because it (i) includes failure rates for each failure mode to achieve a quantitative probabilistic analysis (ii) can be extended to evaluate failure modes that may result in an undesired system state, such as a system hazard, and thereby be used for hazard analysis (Ericson, 2005). The traditional FMEA procedure is summarized as follows (Tay \& Lim, 2006):

(1) Define the scale Table of Severity, Occurrence, and Detect, (2) Studies intent, purpose, goal, and objective of a product/process. Generally, it is identified by interaction among components/process flow diagram followed by task analysis, (3) Identify potential failures of product/process; this includes problems, concerns, and opportunity of improvement, (4) Identify consequence of failures to other components/next processes, operation, customers and government regulations, (5) Identify the potential root cause of potential failures, (6) First level method/procedure to detect/prevent failures of product/process, (7) Severity rating: rank the seriousness of the effect of the potential failures, (8) Occurrence rating: estimation of the frequency for a potential cause of failures, (9) Detect rating: likelihood of the process control to detect a specific root cause of a failure, (10) RPN calculation: product of the three inputs rating; severity, occurrence, detect, (11) Correction. Back to (2) if available, (12) End.

FMEA has been proven to be one of the most important early preventative initiatives during the design stage of a system, product, and process or service (Chin et al., 2008). The crisp RPNs have been considerably criticized for a variety of reasons (Chin et al., 2008; Wang et al., 2009; Chang et al., 2010). However, significant criticisms include but are not limited to the following:

- Different values of $O, S$ and $D$ ratings may produce exactly the same value of RPN, but their hidden risk implications may be totally different. For example, two different events with values of 2, 3, 2 and 4, 1, 3 for $O, S$ and $D$, respectively, will have the same RPN value of 12 . 
- The relative importance among $O, S$ and $D$ is not taken into consideration. The three factors are assumed to have the same importance. This may not be the case when considering a practical application of FMEA.

- The three factors are difficult to precisely determine. Much information in FMEA can be expressed in a linguistic way such as likely, important or very high and soon.

Many decision-making and problem-solving tasks are too complex to be understood quantitatively; however, people succeed by using knowledge that is imprecise rather than precise (Chin et al., 2008). Fuzzy set theory, introduced by Zadeh (1965), is capable of taking into account approximate information and uncertainty to generate decisions. Fuzzy logic was developed later from fuzzy set theory to mathematically represent uncertainty and vagueness and provide formalized tools for dealing with the imprecision intrinsic to many problems (Chin et al., 2008). A framework of a fuzzy FMEA based evaluation approach for evaluating underground mining hazards based on health, safety, and environmental (HSE) is proposed in this paper.

\section{Fuzzy FMEA}

Historically FMEA was in use by NASA as early as 1963 but became better known when implemented by the Ford car manufacturers in about 1977 (Sankar \& Prabhu, 2001). The FMEA is one of the risk analysis approaches recommended by international standards (Dyadem, 2003). Fuzzy FMEA provides a tool that can work in a better way with vague concepts and without sufficient information (Rivera et al., 2009). When there are uncertainties in the relationship among the available criteria or the different options and the relationship cannot be expressed in the form of definitive numbers, the application of Fuzzy theory is useful.

If $\tilde{A}=\left(\mathrm{a}_{1}, \mathrm{a}_{2}, \mathrm{a}_{3}\right)$ is considered as a triangular fuzzy number (TFN), and $\mathrm{a}_{1}, \mathrm{a}_{2}$ and $\mathrm{a}_{3}$ are crisp numbers, and $\mathrm{a}_{1}<\mathrm{a}_{2}<\mathrm{a}_{3}$, then the membership function of $\mathrm{A}\left(f_{(\tilde{A})}(x)\right)$ would be as follows:

$$
f_{(\tilde{A})}(x)=\left\{\begin{array}{cc}
0 & x<a_{1} \\
\left(x-a_{1}\right) /\left(x_{2}-a_{1}\right), & a_{1} \leq x \leq a_{2} \\
\left(a_{3}-x\right) /\left(a_{3}-a_{2}\right), & a_{2} \leq x \leq a_{3} \\
0 & , \\
0 & x a_{3}
\end{array}\right.
$$

Fuzzy FMEA method is used in different researches for evaluating hazards. Chin et al. (2008) proposed a framework of a fuzzy FMEA based evaluation approach for new product concepts. They stated based on the proposed approach and methodologies, a prototype system named EPDS-1, which can assist inexperienced users to perform FMEA analysis for quality and reliability improvement, alternative design evaluation, materials selection, and cost assessment, thus helping to enhance robustness of new products at the conceptual design stage. Xu et al. (2002) presented a fuzzy-logicbased method for FMEA to evaluate engine systems. Tay and Lim (2006) proposed a generic method to simplify the fuzzy logic-based failure mode by reducing the number of rules required by FMEA users for the fuzzy risk priority number (RPN) modeling process. They also evaluated the method using three real-world case studies relating a semiconductor manufacturing process, i.e. test handler process, wafer mounting process, and under fill dispensing process. Wang et al. (2009) carried out risk evaluation in failure mode and effects analysis using fuzzy weighted geometric mean. 
Chang et al. (2010) proposed reprioritization of failures using an intuitionistic fuzzy set ranking technique. They expressed the advantages of the proposed intuitionistic fuzzy set ranking technique are as follows: (1) The proposed method can reduce the occurrence of duplicate RPN numbers, (2) The failure information in FMECA is described as intuitionistic fuzzy variables, which are more realistic and flexible in reflecting real situations, (3) The results of the analysis provide more accurate and effective information for the decision-making process, (4) The proposed approach also provides an evaluation of redundancy place, which can assist the designer in making correct decisions to make a safer and more reliable product design.

Yang et al. (2010) presented a new FMEA model for CNC machine tool using fuzzy theory. Their results indicated that the fuzzy FMEA used in CNC lathe was a reasonable method corresponding to the manufacturing, and it is a validity foundation for constructing reliability design model or supporting control plan of manufacturing. Bukowski and Feliks (2005) proposed method to estimate the risk level for designed system using solutions, which are known from FMEA or FMECA techniques, simultaneously eliminating disadvantages of these methods. They stated algorithms applied in methods FMEA or FMECA helps calculate the value of synthetic coefficient of chance of incident of failure, fuzzy sets were applied to calculating value of this index, and this value is divided into five classes.

$\mathrm{Yu}$ and Skibniewski (2000) described an original solution to continuous constructability knowledge acquisition based on such an approach, combining FMEA with fuzzy knowledge-based systems. Their calculations indicated that FMEA combined with fuzzy knowledge based systems provides systematic approach for acquiring structured and reusable constructability knowledge useful for automated constructability analysis. Tay et al. (2008) presented an FMEA system with a proposed framework equipped with a fuzzy inference system based occurrence model to predict the occurrence score is proposed, and the fuzzy occurrence model is devised.

Liu et al. (2011) presented an FMEA using the fuzzy evidential reasoning (FER) approach and grey theory to improve the effectiveness of the traditional FMEA. Guimarães and Lapa (2004) applied Fuzzy Inference System (FIS) to estimate Fuzzy Risk Priority Number (FRPN) using the expert opinion for quantify linguistic variables. They stated the advantages of the proposed fuzzy rule base for application to FMEA of CVCS can be summarized as follows:

- This fuzzy approach combines (i) expert knowledge and experience for use in an FMEA study, and (ii) can be used for systems where safety data is unavailable or unreliable.

- Converting the scale of RPN traditional in (i) variable linguistic with values defined as input by expert is the great situation and (ii) not force precision and use the system by people without knowledge about interpretations of these linguistic terms. Permitting to use the fuzzy system in a simple way.

- If some change is made in part of system component or sub-component of system analyzed as result of FMEA study, new ranking results after improvements can be obtained so quickly using the "Fuzzy Inference System” (FIS).

\section{Fuzzy Membership Functions}

Severity, Occurrence, and Detect, as in the traditional RPN function, are also used as the input factors for the fuzzy RPN function. The membership functions of these three factors are determined by interpreting the linguistic terms. Tables 1, 2, and 3 summarize some classifications/criteria describing each linguistic term. Fig. 1, Fig. 2, and Fig. 3 depict the membership functions for Severity, Occurrence, Detect, respectively. Output of the fuzzy RPN model, i.e., the RPN value, is divided into 
five equal partitions, namely Low, Low Medium, Medium, High Medium, and High. The membership functions of the linguistic terms are as shown in Tables 5, 6, 7, and 8. The triangular membership function is chosen so as to ensure a smooth transition from one linguistic term to the other.

Table 5

Fuzzy ratings for detection of a failure (Chin et al., 2008; Wang et al., 2009)

\begin{tabular}{ccc}
\hline Rating & Likelihood of detection & Fuzzy number \\
\hline Absolute uncertainty (AU) & No chance & $(9,10,10)$ \\
Very remote (VR) & Very remote chance & $(8,9,10)$ \\
Remote (R) & Remote chance & $(7,8,9)$ \\
Very low (VL) & Very low chance & $(6,7,8)$ \\
Low (L) & Low chance & $(5,6,7)$ \\
Moderate (M) & Moderate chance & $(4,5,6)$ \\
Moderately high (MH) & Moderately high chance & $(3,4,5)$ \\
High (H) & High chance & $(2,3,4)$ \\
Very high (VH) & Very high chance & $(1,2,3)$ \\
Almost certain (AC) & Almost certainty & $(1,1,2)$ \\
\hline
\end{tabular}

\section{Table 6}

Fuzzy ratings for occurrence of a failure (Chin et al., 2008; Wang et al., 2009)

\begin{tabular}{ccc}
\hline Rating & Probability of occurrence & Fuzzy number \\
\hline Very high $(\mathrm{VH})$ & Failure is almost inevitable & $(8,9,10,10)$ \\
High $(\mathrm{H})$ & Repeated failures & $(6,7,8,9)$ \\
Moderate $(\mathrm{M})$ & Occasional failures & $(3,4,6,7)$ \\
Low $(\mathrm{L})$ & Relatively few failures & $(1,2,3,4)$ \\
Remote $(\mathrm{R})$ & Failure is unlikely & $(1,1,2)$ \\
\hline
\end{tabular}

\section{Table 7}

Fuzzy ratings for severity of a failure (Chin et al., 2008; Wang et al., 2009)

\begin{tabular}{lll}
\hline Rating & Severity of effect & Fuzzy number \\
\hline Hazardous without warning (HWOW) & Very high severity ranking without warning & $(9,10,10)$ \\
Hazardous with warning (HWW) & Very high severity ranking with warning & $(8,9,10)$ \\
Very high (VH) & System inoperable with destructive failure & $(7,8,9)$ \\
High (H) & System inoperable with equipment damage & $(6,7,8)$ \\
Moderate (M) & System inoperable with minor damage & $(5,6,7)$ \\
Low (L) & System inoperable without damage & $(4,5,6)$ \\
Very low (VL) & $\begin{array}{l}\text { System operable with significant degradation of } \\
\text { performance }\end{array}$ & $(3,4,5)$ \\
Minor (MR) & $\begin{array}{l}\text { System operable with some degradation of } \\
\text { performance }\end{array}$ & $(2,3,4)$ \\
Very minor (VMR) & System operable with minimal interference & $(1,2,3)$ \\
None (N) & No effect & $(1,1,2)$ \\
\hline
\end{tabular}

\section{Table 8}

Fuzzy weights for the relative importance of risk factors (Chin et al., 2008; Wang et al., 2009)

$\begin{array}{cc}\text { Linguistic term } & \text { Fuzzy number } \\ \text { Very low (VL) } & (0,0,0.25) \\ \text { Low }(\mathrm{L}) & (0,0.25,0.5) \\ \text { Medium }(\mathrm{M}) & (0.25,0.5,0.75) \\ \text { High }(\mathrm{H}) & (0.5,0.75,1) \\ \text { Very high }(\mathrm{VH}) & (0.75,1,1)\end{array}$




\section{Case study}

The method described in the previous section is employed for finding a solution for a real-life problem to reflect the potential application of the proposed model.

Ayerma's Phosphate deposit with an area of about $39 \mathrm{~km}^{2}$ lies on north-west nose of Lar anticline in south-west of Zagros Mountains. Lar Mountains constitutes a NW-SE trending symmetrical anticline including Kazhdumi (Kz), Sarvak (Sa), Gurpi (Gu), Pabdeh (Pa), Asmari (As), Gachsaran (Gs) and Quaternary (Qt) formations. This deposit is situated in about $240 \mathrm{Km}$ north east of Mahshahr port and the biggest sedimentary phosphate reserve in Iran (Fig. 1). This deposit is designed to be extracted by using the Room and Pillar method, one of the most popular underground mining techniques.

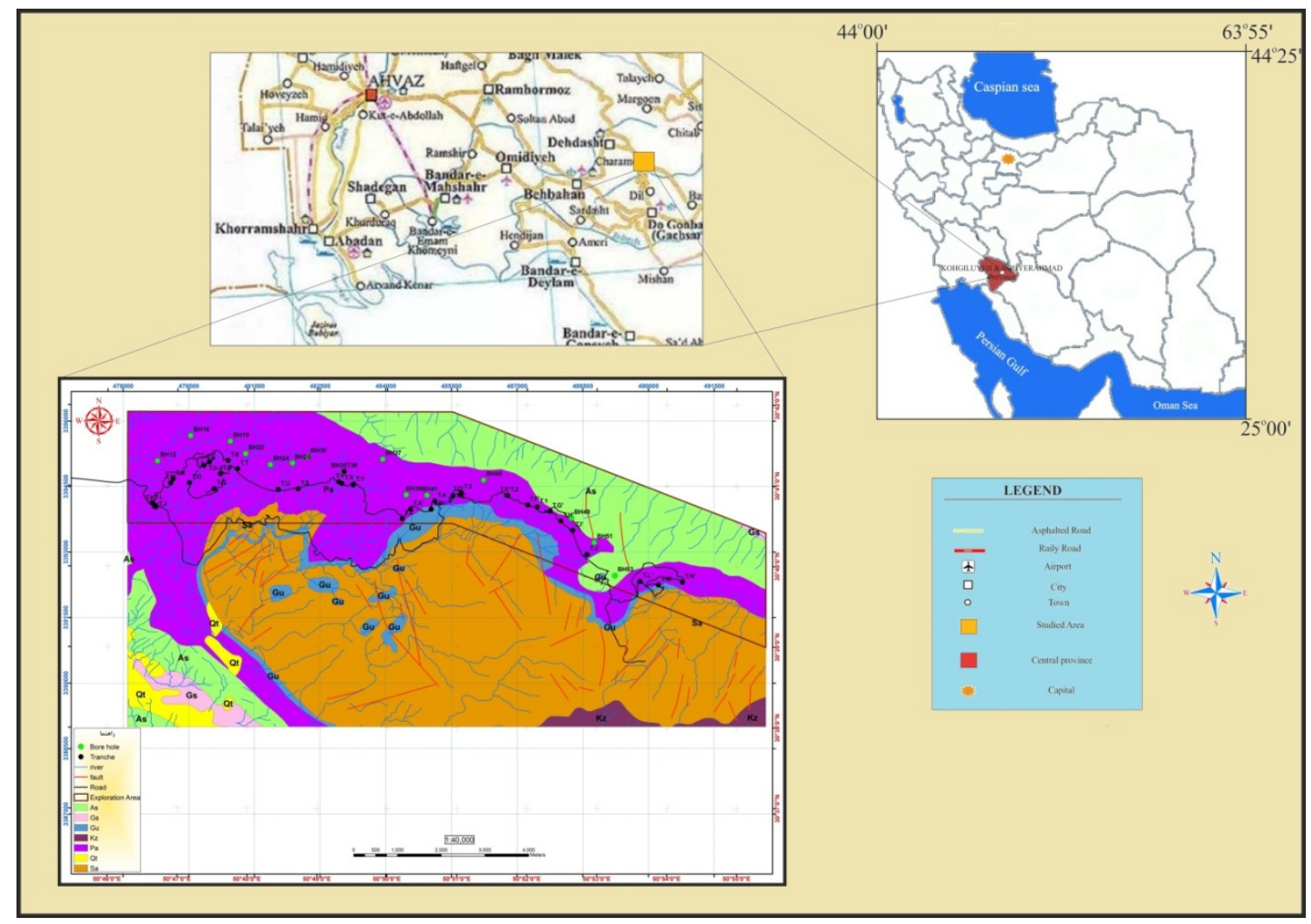

Fig. 1. Geological map of exploration area and the nose parts of Lar Anticline with access to the area

\section{Assessment of underground mining hazards}

Anon (1981) described term hazard as an unsafe situation in a mine. Perception of a hazard is essential because if a hazard is not perceived, no action can be taken to remove it (Ramani, 1992). Finance and time are two components that management always has insufficient access to them. Therefore, management can evaluate hazards and carry out appropriate reaction. Mining operation in general has its hazards. Especially for underground mining has extra dangerous. These hazards are divided to three categories: (i) Health: lake of fresh air, dust, gas, and noisy, (ii) Safety: accident, rock burst, and roof collapse, (iii) Environmental: water pollution, subsidence, and air pollution hazards.

An important contribution of fuzzy system theory is that it provides a systematic procedure for transforming a knowledge base into non-linear mapping. A questionnaire was designed for experts and asked to express their opinions. The fuzzy rule base is generated based on the membership function derived from the experts. Some of these rules can be combined to reduce the number of rules of the fuzzy rule base. However, a total of 205 rules are generated. The rules are written in MATLAB software. The structure of designed system is as Fig. 2. A sample of rules is presented in Fig. 3. A sample of rules is shown as graphically in Fig. 4. 


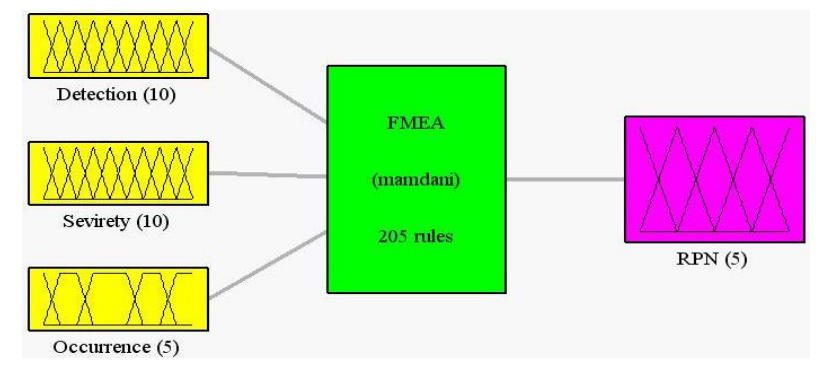

Fig. 2. The structure of designed system

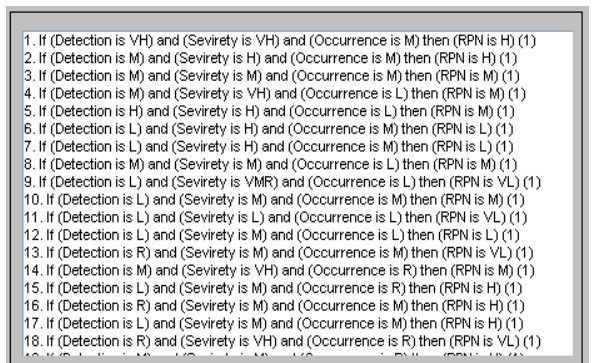

Fig. 3. Sample of rules

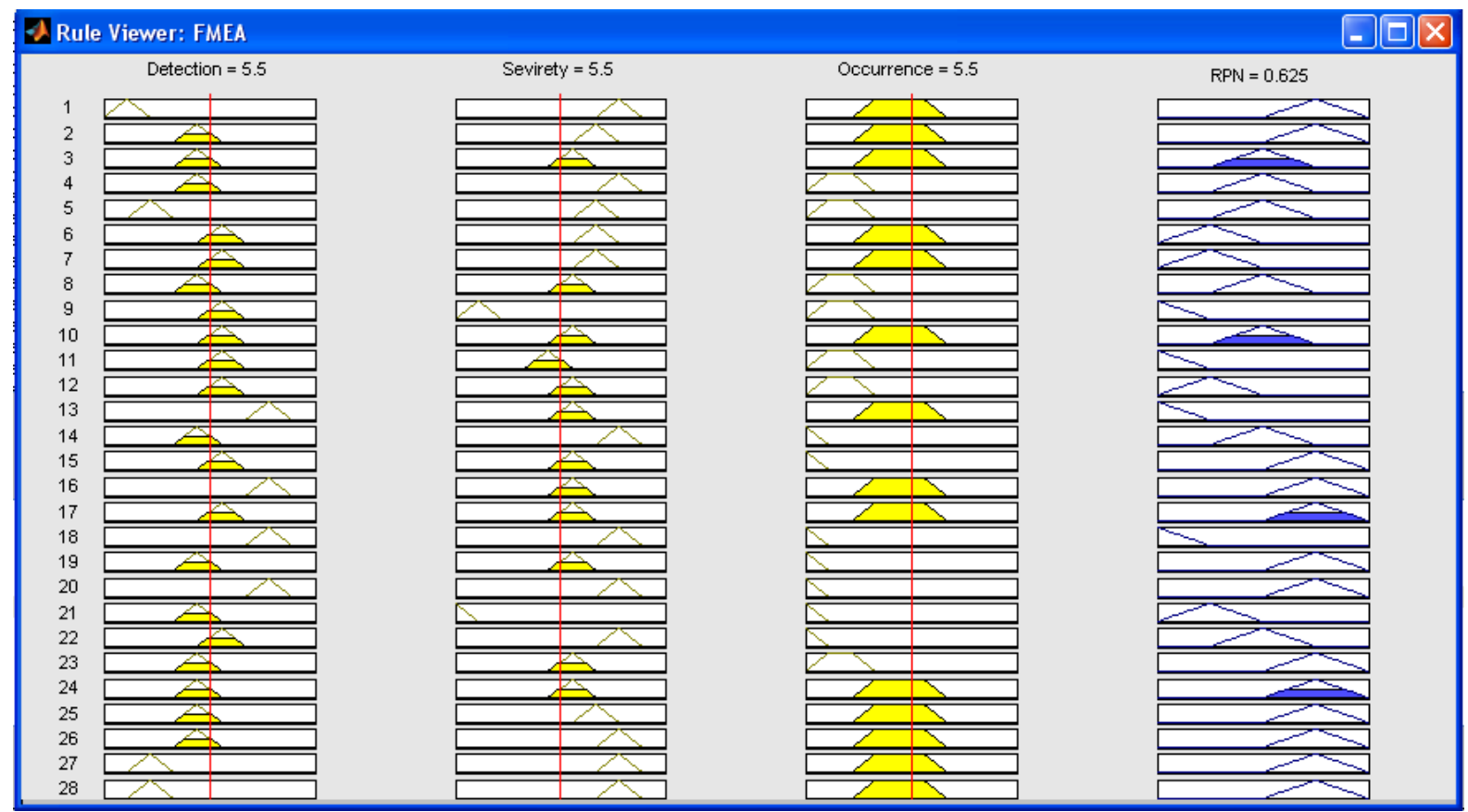

Fig. 4. A sample rule

Then, each hazard is weighted by expert with regard to three risk factors and based on linguistic variables. Table 8 presents the value of expert opinion and the results of network output.

\section{Table 8}

The results of Fuzzy FMEA

\begin{tabular}{|c|c|c|c|c|c|c|}
\hline Criteria & Sub-criteria & Occurrence & Severity & Detection & \multicolumn{2}{|c|}{ Fuzzy FMEA output } \\
\hline \multirow{4}{*}{ 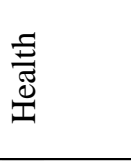 } & Lake of fresh air & $\mathrm{H}$ & $\mathrm{VH}$ & $\mathrm{MH}$ & 0.41 & $\mathrm{M}$ \\
\hline & Dust & M & HWW & MH & 0.56 & M \\
\hline & Gas & $\mathrm{L}$ & HWOW & $\mathrm{H}$ & 0.37 & M-L \\
\hline & Noisy & M & $\mathrm{L}$ & $\mathrm{AC}$ & 0.25 & $\mathrm{~L}$ \\
\hline \multirow{3}{*}{$\underset{\vec{w}}{\overrightarrow{0}}$} & Accident & $\mathrm{M}$ & $\mathrm{VL}$ & $\mathrm{M}$ & 0.53 & $\mathrm{M}$ \\
\hline & Rock burst & $\mathrm{L}$ & HWOW & $\mathrm{L}$ & 0.46 & M \\
\hline & Roof collapse & M & VH & $\mathrm{R}$ & 0.67 & M-H \\
\hline \multirow{3}{*}{ 㽦 } & Water pollution & M & M & $\mathrm{MH}$ & 0.62 & M-H \\
\hline & Subsidence & M & $\mathrm{N}$ & $\mathrm{AC}$ & 0.16 & VL-L \\
\hline & Air pollution & M & MR & $\mathrm{H}$ & 0.34 & M-L \\
\hline
\end{tabular}

Based on the result of the designed system by fuzzy logic in FMEA framework, the most hazardous parameters of Ayerma Phosphate Mine include Roof collapse, Water pollution, dust, Accident, Rock burst, Lake of fresh air, gas, Air pollution, Noisy, and Subsidence, respectively. 


\section{Conclusion}

FMEA is an important technique used to identify and to eliminate known or potential failures to enhance reliability and safety of complex systems and it is intended to provide information for making risk management decisions. In this paper, the author has treated the risk factors $\mathrm{O}, \mathrm{S}$ and $\mathrm{D}$ as fuzzy variables and evaluates them by using fuzzy linguistic terms and fuzzy ratings. As a result, fuzzy risk priority numbers (FRPNs) have been proposed for prioritization of failure modes. The FRPNs have been defined as fuzzy weighted geometric means of the fuzzy ratings for $\mathrm{O}, \mathrm{S}$ and $\mathrm{D}$. For ranking purpose, the FRPNs are defuzzified using centroid defuzzification method. The results indicate Roof collapse is the most hazardous parameter and Water pollution, dust, Accident, Rock burst, Lake of fresh air, gas, Air pollution, Noisy, and Subsidence are in next ranking, respectively.

\section{References}

Bukowski, L., \& Feliks, J. (2005, August). Application of fuzzy sets in evaluation of failure likelihood. In Systems Engineering, 2005. ICSEng 2005. 18th International Conference on (pp. 170-175). IEEE.

Chin, K. S., Chan, A., \& Yang, J. B. (2008). Development of a fuzzy FMEA based product design system. The International Journal of Advanced Manufacturing Technology, 36(7-8), 633-649.

Dyadem, P. (2003). Guidelines for failure mode and effects analysis for automotive. Aerospace and General Manufacturing Industries, Chapter 6, 37-40.

Ericson, C.A. (2005). Hazard Analysis Techniques for System Safety. John Wiley \& Sons, Inc. Chapter 13, 235-259.

Guimarães, A.C.F., \& Lapa, C.M.F. (2004). Fuzzy FMEA applied to PWR chemical and volume control system. Progress in Nuclear Energy, 44(3), 191-213.

Ireson, G., Coombs, W., Clyde, F., \& Richard, Y.M. (1995). Handbook of Reliability Engineering and Management, $2^{\text {nd }}$ ed., McGraw-Hill Professional, New York, NY.

Liu, H. C., Liu, L., Bian, Q. H., Lin, Q. L., Dong, N., \& Xu, P. C. (2011). Failure mode and effects analysis using fuzzy evidential reasoning approach and grey theory. Expert Systems with Applications, 38(4), 4403-4415.

Ramani, R.V. (1992). Personal Health and Safety, SME Mining Engineering Handbook, $2^{\text {nd }}$ ed. Chapter 11.1, 995-1003.

Rivera, S. S., \& Mc Leod, J. E. N. (2009). Recommendations generated about a discontinuous distillation plant of biofuel. In Proceedings of the World Congress on Engineering (Vol. 1, pp. 651-56).

Sankar, N.R., \& Prabhu, B.S. (2001). Application of fuzzy logic to matrix FMECA. Review of Progress in Quantitative Nondestructive Evaluation Vol. 20, ed. by D. O. Thompson and D. E. Chimenti, American Institute of Physics.

Tay, K. M., Teh, C. S., \& Bong, D. (2008, May). Development of a Fuzzy-logic-based Occurrence Updating model for Process FMEA. In Computer and Communication Engineering, 2008. ICCCE 2008. International Conference on(pp. 796-800). IEEE.

Tay, K. M., \& Lim, C. P. (2006). Fuzzy FMEA with a guided rules reduction system for prioritization of failures. International Journal of Quality \& Reliability Management, 23(8), 1047-1066.

Wang, Y. M., Chin, K. S., Poon, G. K. K., \& Yang, J. B. (2009). Risk evaluation in failure mode and effects analysis using fuzzy weighted geometric mean.Expert Systems with Applications, 36(2), 1195-1207.

Winch, G.M. (2002). Managing construction projects: an information processing approach. Oxford: Blackwell Science.

Xu, K., Tang, L. C., Xie, M., Ho, S. L., \& Zhu, M. L. (2002). Fuzzy assessment of FMEA for engine systems. Reliability Engineering \& System Safety, 75(1), 17-29. 
Yang, Z., Xu, B., Chen, F., Hao, Q., Zhu, X., \& Jia, Y. (2010, June). A new failure mode and effects analysis model of CNC machine tool using fuzzy theory. In Information and Automation (ICIA), 2010 IEEE International Conference on (pp. 582-587). IEEE.

Yu,W., \& Skibniewski, M.J. (2000). Continuous constructability knowledge acquisition with fuzzy knowledge-based failure mode and effects analysis, Proceedings of ISARC 2000, Session IT-TE2, Taipei, Taiwan, pp. 1-6.

Zadeh, L. A. (1965). Fuzzy sets. Information and Control, 8, 338-353.

Zeng, J., An, M., \& Smith, N.J. (2007). Application of a fuzzy based decision making methodology to construction project risk assessment. International Journal of Project Management, 25, 589600 . 